\title{
BARRERAS PARA UNA ATENCIÓN EFICAZ EN LOS HOSPITALES DE REFERENCIA DEL MINISTERIO DE SALUD DEL PERÚ: ATENDIENDO PACIENTES EN EL SIGLO XXI CON RECURSOS DEL SIGLO XX
}

\begin{abstract}
Alonso Soto ${ }^{1,2, a}$
RESUMEN

Los hospitales de referencia del Ministerio de Salud peruano atienden a pacientes con patologías complejas. La atención de calidad involucra no sólo el buen trato, la calidez y el humanismo en la atención, sino recursos materiales y capacidad real de resolver problemas. Lamentablemente la realidad de los hospitales del Ministerio de Salud peruano dista mucho de los estándares de calidad recomendados, con notables deficiencias en la capacidad resolutiva debido a la falta de métodos de ayuda diagnóstica, falta de equipamiento y deficiencia en la adquisición de medicamentos e insumos. Es inadmisible que un hospital de nivel referencial no cuente con estudios de imagen como resonancia magnética nuclear o angiografías; estudios de medicina nuclear, pruebas inmunohistoquímicas, estudios de biología molecular, genéticos, entre otros. En este artículo se presentan algunos de los problemas encontrados en la práctica diaria desde la perspectiva del autor como prestador de salud y se revisan los hallazgos de la evaluación de la calidad de atención realizada por la Contraloría General de la República el 2018 en hospitales de referencia. Se proponen algunas medidas inmediatas de acción y se enfatiza la necesidad de abordar de manera inmediata las carencias de infraestructura y capacidad resolutiva para el diagnóstico y tratamiento, así como fomentar los concursos públicos para gestores de hospitales que permitan un ejercicio transparente y meritocrático no sujeto a vaivenes políticos.
\end{abstract}

Palabras clave: Calidad de atención; Equipamiento; Hospital; Sistemas de salud; Equidad en salud (fuente: DeCS BIREME).

\section{BARRIERS TO EFFECTIVE CARE IN THE REFERRAL HOSPITALS OF PERU'S MINISTRY OF HEALTH: SERVING PATIENTS IN THE 21ST CENTURY WITH 20TH CENTURY RESOURCES}

\begin{abstract}
The reference hospitals of the Peruvian Ministry of Health serve patients with complex pathologies. Quality care involves not only good treatment, warmth and humanism in care, but also material resources and real capacity to solve problems. Unfortunately, the reality of the hospitals of the Peruvian Ministry of Health is far from the recommended quality standards, with notable deficiencies in their problem-solving capacity due to the lack of diagnostic aid methods, lack of equipment, and flaws in the acquisition of medications and supplies. It is inadmissible for a referential level hospital not to have imaging studies such as magnetic resonance, nuclear medicine, angiography, immunohistochemistry, molecular biology and genetic studies, among others. This article presents some of the problems encountered in daily practice from the perspective of the author as a healthcare provider and reviews the findings of the quality of care evaluation conducted by the Comptroller General of the Republic in 2018 in reference hospitals. Some immediate measures of action are proposed, emphasizing the need to immediately address the lack of infrastructure and resolution capacity for diagnosis and treatment, as well as to promote public tenders for hospital managers that would allow a transparent and meritocratic exercise not subject to political vagaries.
\end{abstract}

Keywords: Quality of care; Equipment; Hospital; Health systems; Equity in health (source: MeSH NLM).

\footnotetext{
Departamento de Medicina, Hospital Nacional Hipólito Unanue. Lima, Perú

Instituto de Investigación en Ciencias Biomédicas (INICIB), Universidad Ricardo Palma. Lima, Perú

Especialista en Medicina Interna, $\mathrm{PhD}$ en Ciencias Médicas

Recibido: 01/04/2019 Aprobado: 15/05/2019 En línea: 28/06/2019
}

Citar como: Soto A. Barreras para una atención eficaz en los hospitales de referencia del Ministerio de Salud del Perú: atendiendo pacientes en el siglo XXI con recursos del siglo XX. Rev Peru Med Exp Salud Publica. 2019;36(2):304-11. doi: http://dx.doi.org/10.17843/rpmesp.2019.362.4425. 


\section{INTRODUCCIÓN}

El acceso al mejor estándar accesible de salud física y mental es un derecho humano fundamental y un imperativo moral para las naciones ${ }^{(1)}$. Un sistema de salud de calidad y equitativo involucra el fortalecimiento del nivel básico de atención y garantiza que los enfermos que requieren atenciones de mayor complejidad puedan acceder a una atención hospitalaria. Si bien es crucial brindar un especial énfasis a las acciones de prevención, las cuales no sólo involucran al sector salud, sino especialmente al sector educativo (pues es en los niños en quienes se desarrollan los comportamientos y hábitos saludables), la labor esencial de los hospitales es la recuperación de la salud. Para ello es esencial contar con recursos humanos, equipamiento y capacidad de resolver los problemas que aquejan a nuestros pacientes. Esto es especialmente relevante para los establecimientos de mayor nivel de atención, particularmente los hospitales de referencia nacional correspondientes al tercer nivel de atención. Estos hospitales de referencia atienden a pacientes con patologías complejas que muchas veces no pueden ser resueltas por la escasez de recursos con los que deberían contar. Es inadmisible, pero es una realidad cotidiana, que los hospitales de tercer nivel no cuenten con la capacidad de realizar estudios de resonancia magnética, medicina nuclear, angiografías, inmunohistoquímica, estudios de biología molecular o estudios genéticos. Desafortunadamente, las voces de protesta ante tamañas limitaciones son escasas.

La calidad de atención en los hospitales del Ministerio de Salud (MINSA) es percibida como baja (2). El problema no es exclusivo del Perú. La calidad de atención en países de recurso bajos y medianos a nivel mundial es inadecuada pese a que su optimización podría salvar más de ocho millones de vidas anualmente ${ }^{(3)}$. Las muertes por enfermedad cardiovascular tienen la mayor letalidad por la pobre calidad de atención en países de recursos bajos y medios. $Y$ es justamente en esas patologías de creciente incidencia, en donde los estudios diagnósticos y las intervenciones terapéuticas tienen usualmente enormes limitaciones.

Abordaré desde una perspectiva personal, como prestador de salud, las deficiencias percibidas en recursos humanos, equipamiento y medicamentos en hospitales de referencia nacional del Ministerio de Salud peruano. Muchas de las afirmaciones en este artículo surgen a raíz de la experiencia personal durante mis años de trabajo en el MINSA, por lo cual pueden no ser extrapolables a todo el sistema. Sin embargo, considero que pueden ser un punto de inicio para reflexionar sobre nuestras limitaciones y buscar oportunidades de mejora. Trataremos de enfatizar algunas de las oportunidades de mejora que deben abordarse de manera urgente para poder brindar una atención de calidad en los hospitales del MINSA. En la sección final comentaré indicadores y objetivos basados en la mortalidad hospitalaria en salas de medicina y en los resultados de la auditoria de calidad de atención efectuada por la Contraloría General de la República durante el 2018.

\section{RECURSOS HUMANOS: MÁS ALLÁ DE LA FALTA DE PERSONAL}

Es conocido el déficit de recursos humanos del MINSA, con una brecha de más de 47000 profesionales estimada para el $2016^{(4)}$, con un mayor deficiencia en centros de zonas con mayores niveles de pobreza. Las deficiencias en personal técnico son posiblemente mayores, pero poco enfatizadas. En muchos hospitales, la carencia de técnicos que puedan asistir oportunamente a los pacientes en sus necesidades básicas, obliga, muchas veces, a la cooperación de familiares para poder atender este problema.

Paradójicamente, la falta de recursos humanos coexiste con la generación de atenciones innecesarias en las que se utiliza al personal para atenciones que no corresponden al nivel de un centro de referencia. No es razonable que un hospital de referencia cuente con servicios de control prenatal de gestantes sanas o que se realice control de niño sano. Esto evidentemente debe ser realizado en los centros de atención de nivel primario.

La subutilización del recurso humano es también frecuente. Es común el encontrar que los pacientes diabéticos o hipertensos tengan que esperar hasta tres meses para poder obtener una atención en consultorios de endocrinología o cardiología. Y sin embargo los consultorios de medicina interna (que puedan solucionar la mayoría de los casos), no logran completar el total de atenciones posibles con bajas concentraciones de consultas por médico por hora. Lo mismo sucede en los consultorios de pediatría, donde las subespecialidades se ven saturadas de pacientes, mientras que los consultorios de pediatría general podrían tener un mayor número de atenciones.

Finalmente, mientras que la falta de recursos humanos en salud sigue siendo un enorme problema, el exceso de personal administrativo es evidente. Mientras que en Canadá, Uruguay y Paraguay existen 16, cinco y tres médicos por cada trabajador administrativo, respectivamente; en Perú, esta relación incluso se invierte, con tres administrativos por cada médico ${ }^{(5)}$.

Otro problema importante es la subutilización de los espacios físicos de consulta, los cuales podrían emplearse para la atención de pacientes durante la mañana y la tarde. Sin embargo, por regla general, sólo son utilizados durante seis horas diarias. 
La falta de permanencia del personal de salud representa otra limitante para las atenciones médicas. En particular, la consulta externa muchas veces no genera el número de atenciones correspondiente pese a la gran demanda de atención. El rendimiento hora-médico es un indicador importante que debe ser utilizado y monitoreado por los gestores de la consulta externa. El uso de historias clínicas electrónicas que permitan conocer la hora de inicio de atención y número de atenciones brindadas será una herramienta fundamental para cuantificar y monitorizar la consulta externa. La atención paralela de médicos en otras instituciones de salud es una vergüenza que debemos afrontar sin encubrimientos.

Finalmente, no podemos dejar de mencionar la necesidad de mejorar los salarios, fomentar la meritocracia y exigir el cumplimiento eficiente de las responsabilidades del personal de salud para fomentar un sistema más justo con un mejor clima laboral y con una mayor calidad de atención.

\section{AYUDA DIAGNÓSTICA: VOLVIENDO A LOS TIEMPOS HIPOCRÁTICOS}

Los discípulos de Hipócrates eran denominados coprófagos pues utilizaban como métodos diagnósticos el probar el sabor azucarado de la orina del diabético, el sabor acre de las partes necrosadas o el cerumen de los pacientes ${ }^{(6)}$. Estos primitivos métodos han sido (afortunadamente) superados por la disponibilidad actual de métodos de gran precisión. Lamentablemente, la falta de métodos de ayuda diagnóstica constituye una problemática crítica en los hospitales del MINSA y aunque no tengamos que recurrir a los métodos hipocráticos, las carencias nos obligan a realizar diagnósticos con métodos del siglo pasado.

La mayoría de los hospitales operan con tecnología desfasada, lo cual genera retrasos en la calidad de atención. Inexplicablemente no se dispone de algunos exámenes básicos de laboratorio en la casi totalidad de hospitales, tales como cuerpos cetónicos para el diagnóstico de cetoacidosis diabética, antígeno neumocócico en orina, proteinograma electroforético o marcadores específicos para enfermedades autoinmunes. La identificación de enfermedades genéticas es también subóptima, y la mayoría de los hospitales ni siquiera pueden realizar un cariotipo. Todos ello, sumado a la falta de estudios de imágenes, incluyendo resonancia magnética y estudios de medicina nuclear hacen imposible un diagnóstico apropiado, obligando, en muchos casos, a que la familia incurra en gastos catastróficos para realizar, por ejemplo, una resonancia o gammagrafía. Los procedimientos como litotricia, embolización, gamma knife o drenajes percutáneos por radiología intervencionista son extremadamente limitados ya sea por la falta de disponibilidad o por la lentitud e ineficiencia en la compra de insumos.
Un caso emblemático de supuesta negligencia médica lo constituyó, entre otros argumentos, el no colocar un catéter urinario (pigtail) por más de 12 horas en un hospital del seguro social (ESSALUD) ${ }^{(7)}$. En muchos hospitales referenciales del MINSA ese proceso puede durar varios días y hasta semanas dependiendo de la parsimonia para la adquisición de insumos. La situación en hospitales de provincias probablemente sea aún más dramática. Un ejemplo diario que enfrenta el médico de provincias es la escasez de centros de hemodiálisis. Para el 2017, se estimaba que existía un déficit de 300 centros de hemodiálisis ${ }^{\left({ }^{8}\right)}$.

El primer paso para solucionar el enorme déficit en equipamiento hospitalario es cuantificarlo y reconocerlo. ¿Cuál es el déficit de equipos de resonancia magnética, de equipos de litotricia, de equipos de citometría de flujo? Un observatorio que permita monitorizar el equipamiento básico sería un aporte sustancial en tanto sus recomendaciones sean vinculantes. Es necesario formular un petitorio básico de requerimientos de equipamiento para hospitales de referencia a partir del cual se pueda realizar la programación de adquisición de equipos, idealmente a través de compras corporativas que incluyan varios hospitales y que permitan adquisiciones de equipos de alta calidad a precios razonables. En tanto ello ocurre, se debe considerar la tercerización de servicios con criterios definidos y basadas en evidencia para la solicitud de exámenes auxiliares. Es cierto que la tercerización vista como un «negociazo» ${ }^{(9)}$ ha sido origen de actos corruptos por lo cual es mal vista por muchas personas, pero probablemente es una de las pocas soluciones reales en el corto plazo, en tanto se equipa decentemente a los hospitales de referencia. Evidentemente procesos transparentes evitando concertaciones de precios o especificaciones técnicas dirigidas serán necesarios para estas tercerizaciones. La Tabla 1 presenta algunas de las necesidades más urgentes a implementar en la mayoría de los hospitales de referencia peruanos.

\section{FALTA DE MEDICAMENTOS E INSUMOS}

Es común y alarmante la falta de medicamentos básicos incluyendo antihipertensivos, antidiabéticos, antibióticos de amplio espectro, inmunomoduladores y fármacos oncológicos en los diferentes hospitales. Asimismo, la congestión de las salas de operaciones y la imposibilidad de realizar oportunamente procedimientos terapéuticos como colocación de stents, drenajes percutáneos, laparoscopías diagnósticas implican un elevado riesgo de morbimortalidad para los pacientes. Las enfermedades cardiovasculares representan, en este contexto, la mayor carga de muerte por un uso ineficiente de los servicios de salud ${ }^{(3)}$. 
Tabla 1. Algunos métodos de ayuda diagnóstica e intervencionismo que deben implementarse en la mayoría de los hospitales de referencia del Ministerio de Salud

\begin{tabular}{l}
\hline Categorías \\
\hline Patología clínica \\
Marcadores específicos de enfermedades autoinmunes \\
Inmunohistoquímica \\
Citometría de flujo \\
Inmunofluorescencia en tejidos \\
Estudios de biología molecular (reacción en cadena de \\
polimerasa) \\
\hline Estudios genéticos (cariotipo, estudios de mutaciones) \\
\hline Estudios de imágenes \\
Angiotomografía \\
\hline Resonancia magnética \\
Estudios por radioisótopos (gammagrafía, perfusión \\
coronaria) \\
Toracoscopía / Mediastinoscopía \\
\hline Artroscopía \\
\hline Intervenciones terapéuticas \\
\hline Trasplante renal \\
\hline Trasplante cardiaco \\
Trasplante hepático \\
Angioplastia percutánea \\
Embolización terapéutica \\
Circulación extracorpórea \\
\hline
\end{tabular}

\section{GUIAAS CLIINICAS INAPLICABLES POR FALTA DE RECURSOS}

Muchas guías clínicas resultan inaplicables por la falta de recursos materiales y humanos. Esto lleva a la inacción terapéutica. Por ejemplo, la guía nacional de hipertensión arterial en establecimientos de atención primaria plantea el uso del índice tobillo-brazo o evaluación del grosor de la carótida como parte de la evaluación del riesgo cardiovascular ${ }^{(10)}$. Independientemente de lo cuestionable que puedan ser estos procedimientos, no se cuenta con ellos, ni siquiera en muchas instituciones de tercer nivel.

La realización de guías de práctica clínica es una necesidad imperiosa para el Perú. Sin embargo, es irracional solicitar a cada uno de los servicios de cada hospital el plantear guías para sus patologías más frecuentes. Esto resulta en la producción de cientos de guías en su mayor parte inútiles. El recurso humano capacitado apropiadamente para la elaboración de guías es escaso, lo más sensato sería generar equipos multidisciplinarios de varios hospitales para la generación de guías de manejo. Una evaluación de 17 guías de práctica clínica del MINSA encontró que todas tuvieron una baja calidad (11). Recientemente el Instituto Nacional de Salud (INS) ha realizado un convenio con el Seguro Integral de Salud (SIS) para la evaluación de tecnologías sanitarias basadas en evidencia (https://web. ins.gob.pe/es/prensa/noticia/sis-e-ins-firman-conveniopara-mejorar-servicios). Esperemos que ese esfuerzo pueda lograr generar grupos de trabajo interdisciplinarios que convoquen a expertos nacionales y a sociedades científicas para el desarrollo de guías aplicables a todos los establecimientos de mayor nivel de atención.

\section{FALTA DE ATENCIÓN INTERDISCIPLINARIA}

Muchos pacientes necesitan una atención en la que se requiere la toma de decisiones conjuntas entre varios actores y/o servicios. La estructura actual de atención, en la mayoría de los hospitales, hace que la evaluación a través de interconsultas sea tediosa e ineficiente. Un ejemplo clásico lo constituyen los pacientes con diagnóstico de pie diabético hospitalizados en los pabellones de medicina, donde la demora en los procedimientos o intervenciones quirúrgicas usualmente a cargo de los servicios de traumatología, puede llevar a complicaciones graves e incluso a la muerte del paciente. La atención por múltiples especialidades (multidisciplinaria pero no interdisciplinaria) sin una comunicación real entre ellas puede resultar ineficiente y hasta contraproducente. Un caso típico lo constituye el adulto mayor que pasa por diversos consultorios, obteniendo de cada uno, diferentes prescripciones, que sumadas, pueden generar una lista interminable de fármacos con una enorme posibilidad de interacciones y generalmente, un alto gasto de bolsillo.

La solución de este problema involucra un cambio de paradigma en la atención del paciente requiriendo la generación de espacios en los cuales los médicos, de las diversas especialidades, puedan interactuar en forma real para la toma de decisiones conjuntas. Los mecanismos actuales como interconsultas o juntas médicas son claramente ineficientes para este propósito. A nivel hospitalario, la visita conjunta o las juntas médicas programadas son opciones que podrían considerarse.

\section{COBERTURA INSUFICIENTE POR EL SISTEMA DE SALUD}

EI SIS solo atiende a las personas que son categorizadas como pobres o pobres extremos ${ }^{(12)}$. Aunque la evaluación del estado de pobreza es una acción individualizada a nivel municipal, en el Perú se considera que un paciente pobre es aquel que gana menos de 338 soles mensuales (unos 100 dólares) ${ }^{(13)}$. La mayoría de la fuerza laboral peruana está constituida por personas subempleadas que ganan menos del sueldo mínimo, que no aportan a ningún seguro y que, sin embargo, no entran en la categoría de pobreza, pues ganan más de 10 soles ( 3 dólares) diarios. 
Cualquier enfermedad que requiera hospitalización en estos pacientes se convierte en un gasto catastrófico pues sólo el costo del día de hospitalización esta alrededor de seis dólares ${ }^{(14,15)}$ sin considerar medicamentos, insumos o exámenes auxiliares. En muchos de estos pacientes, la atención por falta de recursos se posterga hasta la aparición de complicaciones, en cuyo caso los costos se hacen aún mayores. Un ejemplo dramático de falta de cobertura lo constituyen los 12000 pacientes con insuficiencia renal sin acceso a hemodiálisis ${ }^{(8)}$ no afiliados a ESSALUD y que no son lo «suficientemente pobres» para acceder al SIS. Esto se traduce en un enorme gasto de bolsillo el cual es afrontado, en su mayoría, por personas sin acceso a un seguro de salud. Es inadmisible que nuestro gasto de bolsillo sea más de 200 dólares en comparación, por ejemplo, con Colombia (29 dólares) o Chile (62 dólares) ${ }^{(5)}$.

\section{FALTA DE ATENCIÓN \\ POSHOSPITALARIA DE PACIENTES CON SECUELAS DE ENFERMEDADES GRAVES}

Muchos pacientes que se recuperan de enfermedades graves requieren un cuidado no hospitalario, que es casi imposible de realizarlo en sus domicilios. El ejemplo típico, es el paciente con secuela de desorden cerebrovascular que queda inmovilizado y con requerimiento de sonda nasogástricaeinclusotraqueostomía. Estos pacientestienen un alto riesgo de desarrollar infecciones intrahospitalarias y lesiones por decúbito por lo que su alta pronta es una prioridad. Sin embargo, la familia habitualmente no está en condiciones de brindar un cuidado apropiado por lo que muchas veces permanecen en condición de «hotelería», con el riesgo de morbilidad intrahospitalaria, además de la ocupación de camas que son urgentemente requeridas por otros pacientes. Estos pacientes deberían poder ser institucionalizados temporalmente a fin de completar su recuperación y educar a la familia en el manejo de secreciones, prevención de ulceras de decúbito y manejo general de pacientes dependientes totales. El no contar con este tipo de institucionalización lleva muchas veces a la rehospitalización, particularmente en adultos mayores. Un estudio peruano encontró una tasa de rehospitalización a 30 días del alta, del $14 \%$ en adultos mayores ${ }^{(16)}$.

\section{CARENCIA DE UNIDADES DE TRASPLANTES}

Resulta inexplicable la escasa posibilidad de lograr un trasplante en el MINSA en comparación con ESSALUD. Es injusto que los pacientes del MINSA no puedan tener la esperanza de un trasplante hepático o cardiaco y que sólo uno de sus hospitales logre realizar trasplantes renales. Es una experiencia dolorosa el no poder ofrecer ninguna esperanza de trasplantes a pacientes cirróticos, con neumopatías o cardiópatas avanzados. El ser pobre no debería significar estar condenado a morir por esta carencia del sistema de salud. Se debe enfatizar la necesidad de contar con, al menos, algún centro que pueda ofrecer la posibilidad de trasplantes cardiacos, pulmonares o hepáticos, así como lograr que más hospitales puedan ofrecer la posibilidad de trasplantes renales (Tabla 2).

Tabla 2. Algunos problemas que afectan la calidad de atención en hospitales del Ministerio de Salud

\begin{tabular}{|c|c|}
\hline Problema & Propuesta de abordaje inmediato \\
\hline $\begin{array}{l}\text { Falta de métodos de } \\
\text { ayuda diagnóstica }\end{array}$ & $\begin{array}{l}\text { Equipamiento. En tanto no se } \\
\text { logre el equipamiento necesario } \\
\text { podría tercerizarse en base a } \\
\text { estrictos criterios de necesidad y } \\
\text { transparencia }\end{array}$ \\
\hline $\begin{array}{l}\text { Uso subóptimo de } \\
\text { recursos humanos }\end{array}$ & $\begin{array}{l}\text { Evitar atenciones innecesarias. } \\
\text { Redistribución de atenciones de } \\
\text { especialidades médicas y pediátricas } \\
\text { a servicios de medicina interna y } \\
\text { pediatría general }\end{array}$ \\
\hline $\begin{array}{l}\text { Falta de meritocracia } \\
\text { en la elección de } \\
\text { gestores }\end{array}$ & $\begin{array}{l}\text { Concurso público de méritos para } \\
\text { los cargos correspondientes a la alta } \\
\text { dirección y gestores de hospitales. } \\
\text { Capacitación en administración y } \\
\text { gestión hospitalaria para gestores } \\
\text { como requisito para los puestos } \\
\text { de alta dirección, jefaturas de } \\
\text { departamentos y servicios }\end{array}$ \\
\hline $\begin{array}{l}\text { Cobertura } \\
\text { insuficiente por el } \\
\text { sistema de salud }\end{array}$ & $\begin{array}{l}\text { Aseguramiento de pacientes no } \\
\text { pobres a través de pagos acordes a } \\
\text { su condición socioeconómica. }\end{array}$ \\
\hline $\begin{array}{l}\text { Múltiples guías de } \\
\text { práctica clínica de } \\
\text { baja calidad }\end{array}$ & $\begin{array}{l}\text { Centralizar la elaboración de } \\
\text { guías de práctica clínica a cargo } \\
\text { de unidades de evaluación de } \\
\text { tecnologías sanitarias dependientes } \\
\text { del Ministerio de Salud, Instituto } \\
\text { Nacional de Salud y/o Seguro Social } \\
\text { ESSALUD con colaboración de los } \\
\text { centros de referencia y sociedades } \\
\text { científicas }\end{array}$ \\
\hline $\begin{array}{l}\text { Falta de atención } \\
\text { interdisciplinaria }\end{array}$ & $\begin{array}{l}\text { Normar la atención por equipos } \\
\text { multidisciplinarios para pacientes } \\
\text { con patologías complejas, } \\
\text { particularmente aquellas que } \\
\text { requieran la colaboración } \\
\text { de especialidad clínicas con } \\
\text { especialidades quirúrgicas }\end{array}$ \\
\hline $\begin{array}{l}\text { Falta de atención } \\
\text { poshospitalaria } \\
\text { de pacientes con } \\
\text { secuelas graves de } \\
\text { enfermedad. }\end{array}$ & $\begin{array}{l}\text { Implementación de centros de } \\
\text { institucionalización de pacientes que } \\
\text { requieran cuidados no hospitalarios } \\
\text { (secuelas de enfermedad } \\
\text { cerebrovascular, traqueostomizados) } \\
\text { y atención domiciliaria }\end{array}$ \\
\hline $\begin{array}{l}\text { Falta de información } \\
\text { sobre disponibilidad } \\
\text { de métodos de } \\
\text { ayuda diagnóstica e } \\
\text { insumos }\end{array}$ & $\begin{array}{l}\text { Transparentar en la página web del } \\
\text { Ministerio de Salud y los hospitales } \\
\text { de referencia la cartera de servicios } \\
\text { disponibles en forma real y amigable } \\
\text { para el usuario, y la disponibilidad de } \\
\text { medicamentos e insumos }\end{array}$ \\
\hline $\begin{array}{l}\text { Falta de unidades } \\
\text { de trasplantes }\end{array}$ & $\begin{array}{l}\text { Implementación prioritaria de } \\
\text { unidades de trasplante, capacitación } \\
\text { de los recursos humanos }\end{array}$ \\
\hline
\end{tabular}




\section{UNA MIRADA OBJETIVA A LA CALIDAD DE ATENCIÓN: MORTALIDAD HOSPITALARIA Y EVALUACIONES FORMALES DE CALIDAD DE ATENCIÓN}

\section{MORTALIDAD HOSPITALARIA EN SALAS DE MEDICINA: UN MARCADOR DE CALIDAD DE ATENCIÓN}

Los hospitales del MINSA atienden patologías complejas. Sin embargo, la falta de recursos impide en muchos casos la atención oportuna, lo cual se traduce en altas cifras de mortalidad. Al ingresar al portal del MINSA y a los portales de los distintos hospitales vemos que no se tiene en claro la información o ella se encuentra desactualizada. La tasa de mortalidad hospitalaria ha fluctuado entre el $2,1 \%$ y $2,3 \%$ a nivel global entre 2002 a $2012{ }^{(17)}$. Sin embargo, esta cifra puede no reflejar la situación real dado que en muchos hospitales de referencia los egresos son, en gran parte, por atenciones de parto normal, cesáreas no complicadas (que se atienden por emergencia, dada la dificultad de conseguir salas para operaciones programadas) o cirugías de baja complejidad.

La mortalidad en los servicios de medicina es probablemente un marcador más real de la calidad de atención hospitalaria. Un estudio que analizó la mortalidad hospitalaria en el Hospital Cayetano Heredia entre 1997 y 2008 encontró una mortalidad del 9,6\% en menores de 60 años, la cual se incrementó hasta $14 \%$ en mayores de 60 años ${ }^{(18)}$. En ese mismo hospital la tasa de mortalidad en la década previa había sido del $8 \%{ }^{(19)}$. La situación no es distinta en otros centros hospitalarios. En el Hospital María Auxiliadora la mortalidad en el departamento de medicina durante el 2014 alcanzó el $8,7 \%{ }^{(20)}$, mientras que en el Hospital Nacional Hipólito Unanue durante el 2018 fue del 10,5\% (21). Todas estas cifras son bastante mayores que lo reportados en otros estudios a nivel latinoamericano (22) y global (23). Estos números son alarmantes y ameritan tomar acciones inmediatas.

Se gastan recursos ingentes en evaluar la satisfacción del usuario, lo cual es correcto, pero debe priorizarse el salvar vidas con recursos, medicamentos y capacidad resolutiva que nos permitan un buen diagnóstico y disponer de medicamentos de manera oportuna. Actualmente en la mayoría de los hospitales del MINSA, el realizar una resonancia para un paciente con un tumor cerebral sintomático, conseguir inmunoglobulina humana para un sangrado agudo por trombocitopenia autoinmune o colocar un catéter para nefrostomía de urgencia representan un trámite que puede durar semanas; hacer un recambio valvular de urgencia requiere un trámite de meses. Las carencias de antibióticos para gérmenes drogorresistentes y de agentes biológicos para el tratamiento de enfermedades autoinmunes o neoplasias hacen de estos tratamientos peticiones casi quiméricas. No es de extrañar pues que los pacientes puedan eventualmente complicarse y en el peor de los casos fallecer.

\section{RESULTADOS DE LA EVALUACIÓN DE LA CALIDAD DE} ATENCIÓN SANITARIA EN HOSPITALES PERUANOS

La Contraloría General de la República realizó durante el 2018 una evaluación de la calidad de atención en establecimientos de mediano y alto nivel de complejidad ${ }^{(24)}$. Los resultados han sido poco difundidos, pero por su magnitud requieren una intervención inmediata. Un párrafo sintetiza la situación encontrada:

«Como resultado del operativo se identificó un alto nivel de incumplimiento de las Normas Técnicas de Salud en los servicios de consulta externa, emergencia, farmacia, diagnóstico por imágenes y laboratorio que brindan los hospitales e institutos especializados de salud públicos a nivel nacional, lo que genera el riesgo de una inadecuada atención a los pacientes. Las evidencias de la situación descrita se observan, entre otros, a través de las horas de atención y facilidades de acceso a los pacientes, alta dotación de equipos sin el mantenimiento requerido, elevados niveles de sobre stock de algunos medicamentos y bajo stock de otros, alta exposición a deterioro de los medicamentos por incumplimiento de buenas prácticas de almacenamiento, así como falta de recursos humanos suficientes en los servicios visitados»

Este contundente diagnóstico ameritaría la decisión inmediata de acciones de emergencia. Cabe resaltar que estos datos engloban al MINSA y a ESSALUD, por lo que la situación particular en MINSA es aún más dramática. Solo por citar un ejemplo, al momento de realizar este artículo, ningún hospital de tercer nivel en MINSA contaba con un equipo de resonancia operativo.

La atención en consulta externa también mostró serias deficiencias. El cumplimiento de infraestructura básica para consultorios de medicina interna era sólo del $69 \%$. Por otro lado, al margen de las deficiencias estructurales, no se encontró al internista o cirujano responsable de la atención en el $31 \%$ de casos. La situación en la emergencia no es mejor. El $36 \%$ de los establecimientos de mediana y alta complejidad del MINSA evaluados no contaban con recursos humanos suficientes, el $44 \%$ no contaban con equipamiento adecuado y el $25 \%$ no contaban con infraestructura adecuada. La Tabla 3 muestra un resumen de los resultados del informe presentado por la contraloría.

\section{HOSPITALES Y DIRECTORES: CANTIDAD Y CALIDAD}

Una de las soluciones populistas más recurridas por los gobernantes de turno consiste en la creación de nuevos hospitales. Desde luego es importante contar con un número 
Tabla 3. Características de laboratorio, centros estudios por imágenes y farmacia en establecimientos de atención de mediano y alto nivel de complejidad del Seguro Social ESSALUD, Ministerio de Salud y Fuerzas Armadas en 2018

\begin{tabular}{ll}
\hline Características & Porcentaje (\%) \\
\hline Laboratorio & 37 \\
\hline Recursos humanos insuficientes & 42 \\
\hline No realización de procedimientos mínimos & 42 \\
\hline No cumple con equipamiento mínimo & 27 \\
\hline No cumple con infraestructura & \\
\hline Estudios de imágenes & 53 \\
\hline Infraestructura inadecuada & 68 \\
\hline No cumple con equipamiento mínimo & 45 \\
\hline No licencia de funcionamiento & 53 \\
\hline $\begin{array}{l}\text { No cuenta con ambientes requeridos } \\
\text { No disponibilidad de sala de resonancia } \\
\text { magnética }\end{array}$ & 65 \\
\hline No disponibilidad de sala de angiografía & 39 \\
\hline $\begin{array}{l}\text { No disponibilidad de sala de ecografía de } \\
\text { emergencia }\end{array}$ & 41 \\
\hline Farmacia & \\
\hline Sub stock / desabastecimiento & 42 \\
\hline Prácticas de almacenamiento inadecuadas & 33 \\
\hline Falta de autorización sanitaria del almacén & 45 \\
\hline
\end{tabular}

Basado en los resultados del Operativo de control «Por una salud de calidad» de La Contraloría General de la República en establecimientos de salud de Perú (24)

suficiente de establecimientos para atender a la población. Sin embargo, es importante lograr tener una atención apropiada. Casi ningún hospital del MINSA reúne las condiciones para brindar un diagnóstico óptimo y oportuno para patologías de alta complejidad. En tanto no se disponga de los recursos para atender en forma real a los pacientes continuaremos con estancias hospitalarias prolongadas, complicaciones intrahospitalarias y alta mortalidad. Aunque resulte impopular, probablemente sea más sensato equipar adecuadamente los hospitales existentes, mejorando su eficiencia y reduciendo el número de atenciones de baja complejidad, antes que crear hospitales para un aplauso efímero.

Transparentar los problemas es parte importante en el proceso de toma de decisiones. Ello requiere de autoridades con capacidad real de decisión. Para ello es importante el contar con gestores concursados de modo que puedan tener la autonomía necesaria para afrontar la problemática hospitalaria sin estar sujetos al riesgo de ser removidos de sus cargos de confianza debido a vaivenes políticos o a encarar la problemática hospitalaria de manera firme. Lamentablemente los directores concursados son la excepción y no la regla. De hecho, el contar con gestores formados en Administración y Gerencia Hospitalaria que puedan dirigir los departamentos y servicios hospitalarios es sorprendentemente una utopía que no parece tener una solución inmediata. La capacitación de jefes de departamentos y servicios debe ser una obligación para mantenerse en el cargo.

\section{CONCLUSIÓN}

La falta de recursos en los hospitales de referencia nacional del MINSA constituye una problemática que requiere abordarse de manera inmediata. La carencia de equipamiento, insumos y medicamentos constituye un riesgo para la calidad de atención al paciente. El médico peruano debe ingeniárselas para practicar la medicina en muchos casos con herramientas del siglo pasado. Esto genera una enorme inequidad en la capacidad de resolver patologías de gravedad, particularmente aquella que requieren una atención urgente. La pobreza, la falta de acceso o el acceso inequitativo a la salud y las trabas burocráticas no deberían ser motivos para morir en pleno siglo XXI en un país de recursos medios a altos como el Perú.

Fuente de Financiamiento: Autofinanciado

Conflicto de Interés: El autor no reporta conflictos de interés

\section{REFERENCIAS BIBLIOGRÁFICAS}

1. Office of the United Nations High Commissioner for Human Rights. Committee on economic, social and cultural rights. The right to the highest attainable standard of health: . 11/08/2000. E/C.12/2000/4. (General Comments) [Internet]. Geneva: UN; 2000. [Fecha de acceso 15 Mar 2019]. Disponible en: https://www.nichibenren.or.jp/library/ ja/kokusai/humanrights_library/treaty/ data/CESCR_GC_14e.pdf

2. Pino-Chávez WO. Situación de la calidad de la salud en el Perú. En: Lazo-
Gonzales O, Santivañez-Pimentel A, editores. Atención de Salud Con Calidad [Internet]. Lima: Colegio Médico del Peru; 2018. p. 123-142. [Fecha de acceso 10 mayo 2019]. Disponible en: http://cmplima.org.pe/ wp-content/uploads/2018/06/LibroAtencion-salud-calidad.pdf.

3. Kruk ME, Gage AD, Arsenault C, Jordan K, Leslie HH, Roder-DeWan $\mathrm{S}$, et al. High-quality health systems in the Sustainable Development Goals era: time for a revolution. Lancet
Glob Heal. 2018;6(11):e1196-e1252. d o i : 10 . $1016 / S 2214$. 109X(18)30386-3.

4. Ugarte C. Balances y Retos de los Recursos Humanos en Salud en el Perú [Internet]. Lima: MINSA; 2016 [Fecha de acceso 15 Mar 2019]. Disponible en: https://www.observatoriorh.org/sites/ default/files/webfiles/fulltext/2016/ reu_andino_oct/peru.pdf

5. Sausa M. Situación de la salud en Perú: Ineficiencias del sector están afectando a millones de peruanos [Internet]. 
Perú 21. 2018 [Fecha de acceso 15 Mar 2019]. Disponible en: https:// peru21.pe/peru/situacion-saludperu-ineficiencias-sector-afectandomillones-peruanos-396225

6. Suros A, Suros J. Semiología Médica y Técnica Exploratoria. Octava Edición. Barcelona: Masson; 2001.

7. El Comercio [Internet]. Lima; 2016. [Fecha de acceso 15 Mar 2019]. La tragedia de Shirley: una historia clínica por resolver. Disponible en: https:// elcomercio.pe/lima/tragedia-shirleyhistoria-clinica-resolver-248663

8. Diario Médico [Internet]. Lima; 2017. [Fecha de acceso 15 Mar 2019]. Se necesitan 300 nuevos centros de diálisis en el Perú. Disponible en: http:// diariomedico.pe/impresos/Diario_ Medico_61.pdf.

9. El Comercio [Internet]. Lima; 2016. [Fecha de acceso 15 Mar 2019]. El SIS, el convenio y el 'negociazo' de Carlos Moreno. Disponible en: https:// elcomercio.pe/politica/actualidad/sisconvenio-negociazo-carlos-morenoclaves-399365

10. Ministerio de Salud: Guía de Práctica Clínica para el Diagnóstico, Tratamiento y Control de la Enfermedad Hipertensiva. Lima:MINSA; 2015.

11. Canelo-Aybar C, Balbin G, Perez-Gomez Á, Florez ID. Guías de práctica clínica en el Perú: evaluación de su calidad usando el instrumento AGREE II. Rev Peru Med Exp Salud Publica. 2016;33(4):732. doi:10.17843/rpmesp.2016.334.2559.

12. Ministerio de Salud. SIS Gratuito. 15 de mayo 2013 [Internet]. Lima: MINSA; 2013. [Fecha de acceso 15 Mar 2019] Disponible en: http://www.sis.gob.pe/ Portal/productos/sisgratuito/sis-gratuitoaus/beneficios.html.

13. Instituto Nacional de Estadística e Informática. Resultados De La Pobreza Monetaria 2017 [Internet]. Lima; INEI; 2018. [Fecha de acceso $15 \mathrm{Mar}$ 2019]. Disponible en: https://www. inei.gob.pe/media/cifras_de_pobreza/ presentacion_evolucion-de-pobrezamonetaria-2017.pdf no esta activo

14. Tarifario Hospital Santa Rosa [Internet]. Lima;2019. [Fecha de acceso 15 Mar 2019]. Disponible en: http://190.102.131.45/ transparencia/pdf/2017/tarifario/ TARIFARIO-01-2017.pdf

15. Tarifario del Hospital Hipólito Unanue [Internet]. Lima: Hospital Nacional Hipólito Unanue; 2016. [Fecha de acceso 15 Mar 2019]. Disponible en: http:// www.hnhu.gob.pe/TRANSPARENCIA/ Datos\%20generales/2016/ Resoluciones\%20Directorales/ octubre/486-10-16.pdf

16. Quispe T, Ticse R, Gálvez M, Varela L. Rehospitalización en adultos mayores de un hospital de Lima, Perú. Rev Peru Med Exp Salud Publica 2013;30(4):635-40. doi:10.17843/ rpmesp.2013.304.245.

17. Ministerio de Salud. Compendio Estadístico de Egresos Hospitalarios. Lima: MINSA; 2013. [Fecha de acceso 15 Mar 2019]. Disponible en: http://bvs. minsa.gob.pe/local/MINSA/2723.pdf

18. Tello-Rodríguez T, Varela-Pinedo L, Ortiz-Saavedra J, Chávez- Jimeno $\mathrm{H}$. Estancia hospitalaria y mortalidad en adultos mayores hospitalizados en un hospital general de Lima Metropolitana, 1997-2008. Rev Medica Hered. 2016;22(1):23-8. doi: 10.20453/rmh.v22i1.1096.

19. Fuertes C, Ramos C, Hernandez K, Ramos E, Verdonck K, Chuquiyauri R, et al. Morbilidad y mortalidad en el servicio de hospitalización del Departamento de enfermedades infecciosas, tropicales y dermatológicas del Hospital Nacional Cayetano Heredia entre 1990-2000. Rev Med Hered. 2004 15( 4 ):181-7.

20. Oficina De Estadística e Informática. Anuario estadístico [Internet]. Lima: Hospital María Auxiliadora;2018. [citado 15 de marzo de 2019]. Disponible en: www.minsa.gob.pe/hama

21. Hospital Nacional Hipólito Unanue. Análisis de la Situación de Salud
Hospitalario 2018 [Internet]. Lima: HNHU; 2018 [Fecha de acceso 27 Mar 2019]; disponible en: http://www. hnhu.gob.pe/portal/documentos/wpcontent/uploads/2018/11/RD-23810-18.pdf.

22. Encalada Mora P. Utilidad de las Escalas de (Mpmhos), Rems y Mews para predecir mortalidad general en pacientes hospitalizados en el Servicio De Medicina Interna del Hospital «San Francisco De Quito» [tesis]. Quito: Pontificia Universidad Católica del Ecuador; 2016 [Fecha de acceso 27 Mar 2019]. Disponible en: http:// repositorio.puce.edu.ec/bitstream/ handle/22000/12211/TESIS\%20 COMPLETA\%20ESCALAS\%20 D E \% 20 M O R T A L I A D. pdf ? sequence $=1 \&$ is Allowed $=y$

23. Sanclemente C, Barcons M, Moleiro MA, Alonso F, Pañella D, Carrera R, et al. Mortalidad hospitalaria en un Servicio de Medicina Interna. An Med Interna (Madrid) 2004;21:317-21. doi:10.4321/ s0212-71992004000700002.

24. La Contraloría General de la República. Operativo de control «Por una salud de calidad» [Internet]. Lima: LCGR; 2018. [Fecha de acceso Mar 8 2019]. Disponible en: http:// doc.contraloria.gob.pe/documentos/ operativos/OPERATIVO_POR_ UNA_SALUD_DE_CALIDĀD.pdf

Correspondencia: Alonso Soto

Dirección: Instituto de Investigación en Ciencias Biomédicas (INICIB). Universidad Ricardo Palma. Av. Alfredo Benavides 5440, Santiago de Surco. Lima33. Lima, Perú. Teléfono:996128953

Correo electrónico: sotosolari@gmail.com 